\title{
A Política de Saúde do Idoso sob o espectro CTS: considerações preliminares
}

The Health Policy for the Elderly in the shadow CTS: preliminary considerations

\author{
Meliza Cristina da Silva ${ }^{20}$ \\ Márcia Niituma Ogata ${ }^{21}$ \\ Wilson José Alves Pedro22
}

Artigo recebido em para publicação em out/2013 e aceito para publicação em mai/2014

\section{RESUMO}

O presente artigo tem por objetivo articular aspectos da política de saúde do idoso brasileira ao espectro do campo CTS. Fundamenta a investigação a partir de aportes teóricos de Pierre Bourdieu poder simbólico e campo científico. Considerando-se a necessidade de construção de aportes teóricos e evidências empíricas para a superação das lacunas e obstáculos que permeiam este campo e suas relações com os atores sociais na compreensão dos fenômenos científicotecnológicos, este ensaio de natureza teórica resgata conceitos-chave e analisa algumas diretrizes sobre os processos de envelhecimento brasileiro sob este espectro.

Palavras-chave: Ciência, Tecnologia e Sociedade. Epistemologia, Saúde do idoso.

\section{ABSTRACT}

This article aims to articulate aspects of health policy for the elderly Brazilian to the field spectrum CTS. Based research from theoretical contributions of Pierre Bourdieu - symbolic power and scientific field. Considering the need for construction of theoretical contributions and empirical evidence to overcome the gaps and obstacles that permeate this field and its relations with social actors in understanding some scientific-technological, theoretical nature of this essay rescues key concepts and analyzes some guidelines on the processes of aging in Brazil this spectrum.

Keywords: Science, Technology and Society. Epistemology, Health of the elderly.

\section{INTRODUÇÃO}

Constituído com estudos de enfoque interdisciplinar a partir das décadas de 1960 e 1970, o campo CTS - Ciência Tecnologia e Sociedade investiga os aspectos sociais dos fenômenos científicos e tecnológicos relacionados tanto aos condicionantes sociais, quanto às conseqüenciais sociais e ambientais. Este campo

\footnotetext{
${ }^{20}$ Mestre e doutoranda pelo Programa de Pós Graduação em Ciência, Tecnologia e Sociedade da Universidade Federal de São Carlos, área dimensões sociais da ciência e tecnologia, Universidade Federal de São Carlos. E-mail: meliza@ufscar.br

21 Pós Doutora pelo Programa de Pós Graduação em Enfermagem da Universidade Estadual do Rio de Janeiro, Doutora em Enfermagem, Professora Associada do Departamento de Enfermagem da Universidade Federal de São Carlos, área de saúde coletiva e dimensões sociais da ciência e tecnologia, Universidade Federal de São Carlos. E-mail: ogata@ufscar.br

22 Pós Doutor pelo Instituto do Envelhecimento/ICSS- Universidade de Lisboa, Doutor em Psicologia Social, Professor Adjunto do Departamento de Gerontologia da Universidade Federal de São Carlos, área de gerontologia social e dimensões sociais da ciência e tecnologia, Universidade Federal de São Carlos.E-mail: wilsonpedro@ufscar.br
} 
tem se desenvolvido promovendo a educação científica, favorecendo o desenvolvimento de atitudes e práticas igualitárias com relação às questões sociais e a sua relação com a inovação tecnológica e busca contribuir com a diminuição da distância que se construiu entre a cultura científico-tecnológica e a humana (BAZZO, VON LISINGEN E PEREIRA, 2003).

Defende-se que é preciso construir aportes teóricos e evidências empíricas visando superação das lacunas e obstáculos que permeiam este campo e suas relações com os atores sociais na compreensão dos fenômenos científicotecnológicos. Este é um dos principais objetivos de estudo do CTS e tem nos mobilizado na construção de aportes teóricos e metodológicos que subsidiem estudos relacionados às produções acadêmicas dos Grupos de Pesquisa Políticas e Práticas em Saúde (OGATA et al, 2013) e Núcleo Interdisciplinar de Estudos e Pesquisas em Gerontologia Social ${ }^{23}$ (PEDRO et al, 2013)

A interação destes elementos converge para a constituição de um componente central da cultura, da consciência social e da inteligência coletiva.

Apesar das evidências de melhorias em indicadores de saúde no Brasil (IBGE, 2009) fruto do desenvolvimento científico e tecnológico, bem como dos avanços das políticas de atenção à saúde brasileira, em especial do Sistema Único de Saúde, nas ultimas décadas, muitas lacunas e demandas são evidenciadas. Se tomarmos por análise, as diretrizes sobre os processos de envelhecimento brasileiro sob este espectro, apesar dos avanços das normas e legislações específicas, bem como da inclusão da saúde da pessoa idosa na Agenda Nacional de Prioridade Pesquisa em Saúde (BRASIL, 2008) e do direcionamento de ações estratégicas definidas (BRASIL, 2010) constatam-se paradoxos, contradições e graves implicações.

O presente artigo tem por objetivo articular aspectos da política de saúde do idoso brasileira ao espectro do campo CTS. Fundamenta-se a análise a partir de aportes teóricos de Pierre Bourdieu - poder simbólico e campo científico (BOURDIEU, 2004, 2008, 2011).

23 O Grupo de Estudos Políticas e Práticas em Saúde (GEPPS) prioriza as demandas voltadas para a análise das políticas públicas de saúde, a partir dos pressupostos dos princípios do SUS, dos espaços de saúde, das tecnologias e estratégias de humanização, da educação permanente e da produção científica e tecnológica; e, de outro lado, o Núcleo Interdisciplinar de Estudos e Pesquisas em Gerontologia Social (NIEPGS) que visa aprofundar estudos sobre os aportes teóricos e metodológicos da Gerontologia Social e sobre estratégias, políticas e praticas de promoção do envelhecimento ativo. 
Diversos autores contribuem com reflexões e análises criticas sobre a epistemologia. Optou-se na presente reflexão em priorizar Pierre Bourdieu (2004, 2008, 2011) considerando-se a abrangência e aderência de suas teses com os estudos CTS. Um exercício de reflexão e crítica, relevante no atual estágio da saúde e envelhecimento no âmbito dos estudos CTS.

Os produtos da ciência e da tecnologia são construídos através das demandas sociais, históricas e culturais. A produção técnica e científica pauta-se também em interesses. Estes podem ser públicos e/ou privado, individuais e/ou coletivos, nem sempre éticos e socialmente controlados. Entretanto impactam em toda a sociedade em seu acesso, uso e sustentabilidade. Sua aplicação pode ou não ser benéfica, em seus princípios, meios e fins e em suas múltiplas determinações.

Os estudos CTS em seus paradigmas americano e europeu apontam que os aspectos sociais da ciência e da tecnologia, tanto no que se refere aos fatores sociais que promovem as mudanças científico-tecnológicas (tradição norteamericana), como as consequências sociais e ambientais causadas pelo desenvolvimento científico-tecnológico (tradição europeia) (BAZZO, VON LISINGER E PEREIRA, 2003).

Tais interações demonstram que todo desenvolvimento científico-tecnológico é social e historicamente construído e tem consequências nem sempre positivas que afetam a sociedade. Tal como Sousa-Santos nos aponta é preciso "um conhecimento prudente para uma vida decente" (SANTOS, 1996).

Na América Latina, o chamado Pensamento Latino Americano de Ciência, Tecnologia e Sociedade - PLACTS concentra-se em estudos voltados para o contexto regional e seu processo de desenvolvimento, através da ênfase nas políticas públicas envolvendo ciência e tecnologia e os processos de participação pública em ciência e tecnologia. Nesta perspectiva propõe-se compreender algumas dimensões científicas e tecnológicas da saúde do idoso em seu caráter regional.

\section{APORTES TEÓRICOS}

Dentre as contribuições do pensamento de Pierre Bourdieu, duas dimensões serão priorizadas para análise da problemática em questão: a dimensão do poder simbólico e campo científico. Ambas carecem de um enquadre dada sua 
complementariedade e elucidam a tese: "qualquer que seja o campo, ele é objeto de luta tanto em sua representação quanto em sua realidade" (BOURDIEU, 2004, p.29). Analisadas num contexto macro:

\begin{abstract}
se fosse preciso realçar uma característica original da sociologia francesa do período entre 1945-1990, seria tentador sublinhar o interesse particular em repensar tudo o que está relacionado com os sistemas simbólicos no seu sentido mais amplo. Muito mais do que outras correntes anteriores, estas perspectivas sociológicas estiveram atentas a tudo o que respeita às linguagens, aos sistemas de representações, aos símbolos, às crenças, às ideologias (ANSART, 2002, p.121).
\end{abstract}

E é neste período que se localiza Pierre Bourdieu (1930-2002), que ingressou na École Normale Supérieure, em 1951, em Paris, tendo se graduado em 1954. Foi professor de liceu entre 1955 e 1958. Viveu na Árgelia, entre 1958 e 1962, um período marcado pela guerra colonial. Nesta fase trabalhou e realizou estudos etnográficos considerados marcantes em sua trajetória intelectual, em especial os estudos sobre a antiga Cabília que introduz a criação de suas reflexões sobre a problemática das práticas simbólicas.

Em seu retorno à França, trabalhou nas Universidades de Paris e de Lille. Foi Diretor de Estudos da École Pratique dês Haus Études, em 1964. Dirigiu o Centre de Sociologie Européenne, a partir de 1968. Fundou a revista Actes de La recherche em sciences sociales, em 1975. Foi responsável pela cadeira de Sociologia no Collège de France, desde 1981.

Estas vinculações atreladas à sua produção acadêmica de centenas de estudos, publicados em livros e artigos, caracterizam um legado do autor, que expressa uma importante vertente da sociologia francesa contemporânea. A análise das práticas simbólicas nas suas diversas dimensões, contempladas nos estudos sobre estudantes, as práticas artísticas, a distinção e a universalidade estão contemplados no pensamento de Bourdieu: um filósofo? Um sociólogo?

A noção de campo se constitui numa das importantes contribuições deste autor para o foco do presente estudo. Os campos são os espaços onde atores sociais se posicionam de acordo com o capital acumulado e poder simbólico manifesto. Manifestam-se nas várias dimensões sociais: científica, jurídica, cultural e econômica. Designam espaços relativamente autônomos, já que são compostos por especialistas em determinados assuntos. A avaliação é feita por seus pares conforme os interesses do campo. 
Os campos são os lugares de relação de forças que implicam tendências imanentes e probabilidades objetivas. Um campo não se orienta totalmente ao acaso. Nem tudo nele é igualmente possível ou impossível em cada momento. Entre as vantagens sociais daqueles que nasceram num campo, esta precisamente o fato de ter, por uma espécie de ciência infusa, o domínio das leis imanentes do campo leis não escritas que são inscritas na realidade em estado de tendências e de terma o que se chama em rugby, mas também na Bolsa, o sentido do jogo ${ }^{24}$. (BOURDIEU, 2004, p.27)

Estes campos são definidos pelo conjunto de valores que definem o senso comum destes sujeitos. Nestes espaços são definidas regras que selecionam os participantes conforme interesses e créditos valorizados pelo grupo.

\begin{abstract}
Num estado de campo em que se vê o poder por toda parte, como em outros tempo não se queria reconhecê-lo nas situações em que ele entrava pelos olhos dentro, não é inútil lembrar que - sem nunca fazer dele, numa outra maneira de o dissolver, uma espécie de "circulo cujo centro está em toda a parte e em parte alguma" - é necessário saber descobri-lo onde ele se deixa ver menos, onde ele é mais completamente ignorado, portanto, reconhecido: o poder simbólico, é, com efeito, esse poder invisível o qual só pode ser exercido com cumplicidade daqueles que não querem saber que Ihe estão sujeitos ou mesmo que o exercem" (BOURDIEU, 2011, p.4)
\end{abstract}

A noção de campo científico apresentado por Bourdieu (2004) é de que para compreender uma produção cultural - científica ou literária - não basta referir-se ao conteúdo textual e social desta produção. Entre o texto e o contexto há um universo intermediário que nomeia respectivamente como literário, artístico, jurídico ou científico.

Neste campo estão os agentes que produzem ou reproduzem a literatura, a arte ou a ciência, conforme as leis sociais deste universo.

Para estar em um campo cientifico é preciso atender aos requisitos de seleção deste campo, requisitos de formação, de poder social ou financeiro, ou ainda outros interesses comuns ao grupo.

\title{
SAÚDE DO IDOSO E O CAMPO CTS: APROXIMAÇÕES
}

A transição demográfica tem movimentado a sociedade na construção de políticas sociais e de saúde voltadas ao idoso. Grandes são os desafios, em especial a partir da Constituição Federal do Brasil de 1988 e seus desdobramentos em diversos estatutos jurídicos para o segmento populacional que envelhece, garantindo direitos à população e obrigações ao Estado. Destaque merece a criação do Sistema Único de Saúde (Lei 8080/90 e 8142/90), a Política Nacional do Idoso

\footnotetext{
${ }^{24}$ Sens du placement, no original (N.T.).
} 
(Lei 8842/94) e o Estatuto do Idoso (Lei 10741/2003), dentre outros estatutos que garantem direitos os direitos da população brasileira, tendo a saúde em conceito latu. A ampliação da expectativa de vida é também fruto dos avanços científicos e tecnológicos, bem como das mudanças de estilo de vida do cidadão.

Desde 2002 por ocasião da Segunda Assembléia Mundial das Nações Unidas, realizada em Madri, defende-se a emergência da prioridade do Envelhecimento Ativo, definido como um "processo de otimização das oportunidades de saúde, participação e segurança, com o objetivo de melhorar a qualidade de vida à medida que as pessoas ficam mais velhas" (WHO, 2005, p. 13).

De acordo com Carneiro et al. (2013), em alguns países os gastos com saúde desta parcela da população que possuem mais de 65 anos giram em torno de 40 a $50 \%$ e os custos per capita é de 3 a 5 vezes maior que das outras faixas etárias.

Segundo informações da União Nacional das Instituições de Autogestão em Saúde - UNIDAS (2011, apud CARNEIRO et al, 2013), embora não haja estimativa do gasto público total por faixa etária da população, o gasto médio representa 7,5 vezes comparado a despesa média verificada para a primeira faixa etária ( 0 a 18 anos).

No Brasil foi realizada uma estimativa a partir de dados de internação no SUS e detectou-se que 54,9\% da diferença esperada nos gastos entre 2004 e 2050 são explicados pela mudança na estrutura etária nesse período (BERENSTEIN; WAJNMAN, 2008 apud CARNEIRO et al, 2013).

Segundo Pedro (2013), as políticas públicas são produtos das lutas e negociações da sociedade civil como um todo. Entretanto, no pano de fundo encontram-se evidências de interesses articulados com objetivos específicos, sejam eles partidários, econômicos ou pessoais, que merecem problematização.

As ciências aplicadas na gestão da saúde, e por extensão ao âmbito das investigações e intervenções no âmbito do envelhecimento são resultados de escolhas e negociações. As políticas públicas determinam as ações do público e do privado.

Não há forma de pensar saúde em um território sem este envolvimento, pois o equilíbrio econômico para o financiamento da saúde no Brasil conta com a participação de ambos os setores. 
O Estado de São Paulo, em sintonia com o Pacto pela Saúde (BRASIL, 2006) está organizado em subdivisões regionais visando dentre suas atribuições desenvolver ações de educação permanente e fomentar o controle e a participação social, nos diversos segmentos: trabalhadores da saúde, gestores municipais e estaduais, organizações públicas e privadas de saúde e a sociedade.

Tal como Bourdieu afirma, problematizamos: como estes espaços são construídos? Qual a natureza deste campo? Que poder simbólico se manifesta? Como o envelhecimento populacional transforma as relações e demandas da sociedade com os serviços de saúde pública? À medida que a transição demográfica brasileira avança, o perfil epidemiológico e econômico na saúde também passa por transformações: que impactos estas evidenciam no campo CTS?

Em estudo proposto para o campo CTS (SILVA, OGATA e PEDRO, 2013) estabelece-se como recorte a análise das práticas dos articuladores da saúde dos idosos e dos gestores municipais de saúde por representarem o Sistema Único de Saúde Brasileiro (SUS) em sua localidade e pela sua participação na implementação de políticas públicas.

Os articuladores da saúde do idoso são selecionados através de indicação dos municípios. Geralmente são profissionais que atuam na temática, seja na atenção básica, ou mesmo na gestão das secretarias municipais de saúde. A proposta é construir através da educação permanente em saúde, uma rede interdisciplinar e intersetorial de qualificação de trabalhadores para o desenvolvimento de ações de prevenção, promoção e reabilitação em saúde nas demandas do envelhecimento. É também objetivo desse formato, o desenvolvimento de ações que promovam o envelhecimento em convergência com a política de saúde do idoso em todas as esferas públicas, tal como a Organização Mundial de Saúde preconiza.

Na maioria das vezes, os gestores municipais de saúde são atores escolhidos pelo prefeito para os chamados "cargos de confiança". Para este ator recomendamse habilidades técnicas, conceituais e humanas, para que exerça o cargo, em sintonia com as diretrizes do SUS. Estes gestores representam de um lado os interesses do poder público, cujas ações variam conforme o legislativo e os planos de governo e de outro os interesses da sociedade. 
Constata-se uma possível divergência de interesses e opiniões, que demandam negociações e acordos entre esses atores na implementação da Política de Saúde do Idoso. Neste sentido, a nossa aproximação dos conceitos de capital social e cientifico definidos por Bourdieu na busca da compreensão desse contexto.

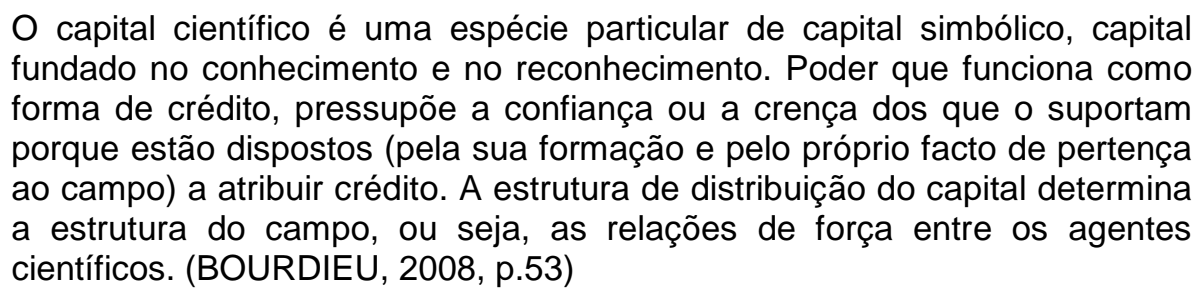

Os créditos são definidos pelo capital, que em determinados contextos pode ser cientifico, econômico ou social. A credibilidade e o poder de implementação de uma ideia, ação ou política pública sofre influencia deste capital e dos interesses do campo.

Para Bourdieu (2008, p. 55), o espaço de manobra deixado às estratégias dependerá da estrutura do campo, caracterizada por um nível mais ou menos elevado de concentração de capital. Neste contexto, os detentores de maior capital são os responsáveis por ditar as regras do jogo.

O campo de estudos do envelhecimento, com prioridade na saúde, assim como os demais campos científicos possuem regras e uma estrutura de capital. Tudo é negociado, mesmo os detentores de maior credibilidade precisam se submeter às relações políticas quando tratamos de questões relacionadas a políticas publicas ou a ações que movimentem a estrutura social de uma localidade. A decisão de envolver ou não o setor privado, de implementar ou não determinada ação são questões que compõem o jogo de negociação no campo.

Para Bourdieu (2008), o espaço de manobra deixado às estratégias dependerá da estrutura do campo, caracterizada, por exemplo, por um nível mais ou menos elevado de concentração do capital. Existem os dominantes e os dominados, os primeiros em posição de impor a representação da ciência conforme seus interesses, ou seja, eles definem a forma de jogar e as regras do jogo.

No caso da saúde do idoso, o campo precisa cumprir o requisito da transparência. A participação de caráter deliberativo de representantes de usuários dos serviços de saúde nos conselho municipal do idoso e conselho municipal da saúde responde pelo controle social nessas áreas. Uma das questões a ser 
levantada refere-se à forma como é conduzida a discussão e o efetivo conhecimento que a sociedade possui a cerca da temática antes da validação das estratégias e propostas que futuramente serão as políticas públicas do município, região e Estado.

O exercício de compreender a temática, as relações e as estratégias de atuação do campo fornecem subsídios para interagir ou mesmo participar do processo. Para se jogar o jogo e exercer influencia sobre ele, é preciso conhecer o jogo.

A sociedade carece de estratégias provenientes da Ciência e Tecnologia que possibilitem a ampliação de discussões relacionadas à saúde do idoso.

A sociedade precisa compreender melhor a mudança demográfica e os impactos que estão por vir e o resgate da cidadania na participação pública é fundamental para construção de políticas publicas de saúde do idoso.

O campo científico do envelhecimento no espectro da saúde é composto por agentes das diversas áreas que estabelecem entre si níveis de capital cientifico diferentes e um conjunto de interesses diversos. Como conciliar estes conhecimentos em prol de interesses comuns, voltados para o bem social?

A dimensão que este campo alcança e os impactos que emite em outras áreas como educação, assistência social e na economia pode acontecer em grandes proporções.

\section{CONSIDERAÇÕES FINAIS}

A aderência do pensamento de Pierre Bourdieu ao campo CTS aponta uma possibilidade de aplicação da educação CTS, bem como a expectativa de ampliação do conceito de cidadania e o incentivo da conscientização para a participação pública em assuntos relacionados com a ciência e a tecnologia.

Estudos como o de Carneiro (2013) apontam para um crescente aumento de custos resultante da mudança demográfica e do perfil epidemiológico brasileiro em um futuro não tão distante. Projeções do Instituto Brasileiro de Geografia e Estatística - IBGE apontam mudanças substantivas para as próximas décadas (IBGE, 2013). O fato é que a transformação ocorrerá cedo ou tarde e a Gestão Publica deve iniciar o processo articulado de apropriação e divulgação do conhecimento científico, de inovação tecnológica e de gestão de ações, no sentido de preparar a sociedade e os serviços para esta nova demanda e cultura. 
Portanto, a política de saúde do idoso como objeto de estudo evidencia grande complexidade, quer seja na perspectiva das representações sociais ou à luz dos aportes teóricos de Bourdieu $(2004,2008,2011)$ sobre o poder simbólico e campo cientifico, destacando os jogos de interesse e as negociações entre os atores sociais envolvidos. Sua magnitude só pode ser compreendida através de uma perspectiva interdisciplinar, conforme se configura o campo CTS.

Neste espectro, podemos observar o quanto o campo do envelhecimento pode ser determinado pelos interesses e crédito dos atores envolvidos, direcionados ou não para a sociedade. A reflexão acerca destas questões consistem em importante contribuição para o estudo da política de saúde do idoso brasileiro.

\section{REFERÊNCIAS}

ANSART, P. Sociologia francesa contemporânea. Trad. Rodrigo Rosa. Celta, Oeiras, 2002.

BAZZO, W.A; VON LINSINGEN, I.; PEREIRA, L.T.V. Introdução aos estudos CTS (Ciência, Tecnologia e Sociedade). Madri: Cadernos de Ibero-América, 2003.

BRASIL. Lei 10.741, de 1 de outubro de 2003. BRASIL, 2003.

BRASIL. Lei 8.080, de 19 de setembro de 1990. BRASIL, 1990.

BRASIL. Lei 8142, de 28 de dezembro de 1990. BRASIL, 1990.

BRASIL. Lei 8842, de 04 de janeiro de 1994. BRASIL, 1994

BRASIL. Portaria № 2.528 de 19 de outubro de 2006. BRASIL, 2006.

BRASIL. Agenda Nacional de Prioridade de Pesquisa em Saúde. Ministério da Saúde. Secretaria de Ciência, Tecnologia e Insumos Estratégicos. Departamento de Ciência e Tecnologia. Brasília, Ministério da Saúde, 2008.

BRASIL. Atenção à Saúde da Pessoa Idosa e Envelhecimento. Ministério da Saúde, Brasilia, DF, 2010.

Indicadores Sociais de Saúde e Demográfico. Rio de Janeiro. Instituto Brasileiro de Geografia e Estatística, 2009.

Instituto Brasileiro de Geografia e Estatísticas, 2013. www.ibge.org.br <acesso em 11 de outubro de 2013>

BOURDIEU, P. Os usos sociais da Ciência- Por uma sociologia clínica do campo cientifico. São Paulo: Fundação Editora da UNESP, 2004. 
BOURDIEU, P. Um mundo a parte. In: Para uma sociologia da Ciência. Lisboa: Edições 70, 2008.

BOURDIEU, P. O poder simbólico. Lisboa, Edições 70, 2011.

CARNEIRO, L. A. F. et al. Envelhecimento populacional e os desafios para o sistema de saúde brasileiro. São Paulo: IESS, 2013. Disponível em: http://www.iess.org.br/envelhecimentopop2013.pdf. Acesso em: 01 de maio de 2013.

OGATA, M.N. et al. Grupo de Pesquisa Políticas e Praticas de Saúde. Anais da Jornada Científica da UFSCar, Workshop de Grupos de Pesquisa. 2013.

PEDRO, W.J.A. et al. Núcleo Interdisciplinar de Estudos e Pesquisas em Gerontologia Social. Anais da Jornada Científica da UFSCar, Workshop de Grupos de Pesquisa. 2013.

SANTOS, B.S. Um discurso sobre as ciências. $8^{\mathrm{a}}$ Ed. Porto, Portugal, Afrontamento, 1996.

SILVA, M.C; OGATA, M.N; PEDRO, W.J.A. As representações sociais do envelhecimento na perspectiva da ciência, tecnologia e inovação na saúde. 2013. Projeto de Pesquisa Cientifica (Doutorado)- Universidade Federal de São Carlos, São Carlos, 2013.

WHO. Envelhecimento ativo: uma política de saúde. World Health Organization; trad. Suzana Gontijo. Brasília, Organização Pan-Americana de Saúde, 2005. 
even under the natural appearance of limits, they are natural references (geographic forms), don't let pass as a socio historical translation.

We could also affirm that the reality here is the expression and the translation of who live in the studied territory, study that developed by collective methodologies that aimed to break, or even attenuate, the vertical relation that characterize the science in its dichotomy, between the research object and the subject. We worked to elaborate a study in which the construction/translation of 'reality' was not only produced by the researchers (dominant perspective), but like a conjunction of perspectives, represented by the researchers together with the people that participated as subject of their own process.

Our simple experience to produce science in a participative way, in our point of view, try to dialog with the Bruno Latour (2001) observations, principally breaking the knowledge perception funded into the dualism represented in the expressions: subject/object and watcher/watched. This duality should be thought as necessary to the science rationality, because should be as corollary for the whole translation of one reality. Contrarily, our work tried to break this duality, but even doing so, could we get the whole reality translation, or the closest from what we could call of truth?

Bruno Latour (2001) comments about the use of maps in scientific researches, and how the scientist dominate the world; however this same world has to be translated into concepts and forms: yes, the scientists dominate the world - but since the world came until them in the form of bidirectional inscriptions, super posts and combined. If this happens with the science production, we perceive that the same happen with the ethno maps, since the references that constitute the drawn and the map are social representations that the Pataxó people do of their territory, there are codes, concepts and inscriptions that mean the living world and its spatiality.

However we find out that the ethno map Pataxó produced (even elaborated from the Pataxó point of view), was favored by the knowledge and instruments elaborated from other disciplines areas represented on the use do GPS and GIS. So, the georeferenced positions are well accepted because the precision that the equipments are known to have, but even in face of this aspect of precision available in the market, they don't spoken about the world, but build representations that sometime are pushed far way, other time approached. In the same way occur with the Pataxó's representation about their territory, when they draw their community, 
their representation, theydon't talk about the own world, but about the world as a stage of a theater where those representation are realized.

\section{CONCLUSIONS}

In this sense, we also observed the difference of the maps made without the participation of its actors, which consists in a representation of certain space determined by the community as important and not the whole space or the space determined for someone else. The confection of the ethno maps and the discussion of them generated reflections regarding the local reality. Were thought situations from current time, that deviate of the old scenario, as change in the cultivation system, the growth number of the families, and the amount and water quality.

The reflections about the space, in turn, facilitated the perception of vulnerability factors and of measures that are, or are not being taken to regarding them. In that sense, was possible to meditate as expected, the cultural, social, political and environmental aspects for the social technology construction.

In this context the digital technology is indispensable and the resources, limited. Still, the used resources were understood by the community that participated in the whole process. However, although it is still restricted the access to instruments (GPS) and to computer programs for mapping, the activity is viable for indigenous communities. The indigenous, like most Brazilians, have easiness using cell phones or devices like music and video players, that don't differ a lot in commands and handling, such like a standard GPS.

We noticed that is possible to promote the exercise of political power by local traditional people, to lead their cultural diversity and biodiversity conservation. It is important to point out that the community development in this process can be stimulated by the construction of autonomy and empowerment of those traditional people.

\section{Acknowledgements}

To the all Imbiruçu indigenous for the support in this study. Especially to the Cacique Romildo Pataxó and the Pataxó students Keyla Francis de Jesus da Conceição and Wakey Silva dos Santos (Junior Scientific Initiation Scholarship, UFVJM). To the Fundação de Amparo a Pesquisa do Estado de Minas Gerais (Fapemig) and to the National Council for Scientific and Technological Development (CNPq), for the scholarships. 


\section{REFERENCES}

ANGTHICHAY, ARARIBY, JASSANÃ, MANGUAHÃ, \& KANÁTYO. O povo Pataxó e suas histórias. 6.ed. São Paulo: Global, 47p., 2002.

BLACK, J. Mapas e história: construindo imagens do passado. 1.ed. Bauru, SP: EDUSC, 428p., 2005.

CAMPOS, R.C. Movimentos indígenas por educação: novos sujeitos socioculturais na história recente do Brasil. In: Reunião Anual da ANPED, n.23, Caxambu, 2000.

CARVALHO, A. $\mathbf{O}$ geoprocessamento na gestão ambiental em Terras Indígenas: uma experiência com etnomapeamento junto à Comissão Pró-Índio do Acre. Science Master Dissertation (Physical Geography). Geography Department, Faculdade de Filosofia, Letras e Ciências Humanas, USP, São Paulo, 127p., 2006.

CEDEFES. Centro de Documentação Eloy Ferreira da Silva. A luta dos índios pela terra: Contribuição a história indígena de Minas Gerais. 1.ed. Contagem, 120p., 1987.

CEDEFES. Centro de Documentação Eloy Ferreira da Silva. (2006). Pataxó: Quem é esse povo? Disponível em: $<$ http://www.cedefes.org.br/new/index.php?conteudo=materias/index\&secao=1\&tema $=53 \&$ materia $=1774>$ Acesso em: 15/06/2010.

CHAMBERS, R. The origins and practice of participatory rural appraisal. World Development, v. 22, n. 7, p. 953-969, 1994.

CHAVES, A. S.; COLLI, F. Processos educativos Revista Terra Viva: estratégias, ações, resultados e desafios na disseminação agroflorestal. Brasília: Ministério do Meio Ambiente. Série Sistematização v. 1, p. 22-33, 2006.

CORREIA, C. S. Etnozoneamento, etnomapeamento e diagnóstico etno ambiental: representações cartográficas e gestão territorial em Terras Indígenas no Estado do Acre. PhD Thesis (Antropology) - Antropology Department, UnB, Brasília, 420p., 2007.

FARIA, A. A. C. O uso do diagnóstico rural participativo em processos de desenvolvimento local: um estudo de caso. Dissertation (Master Science) Universidade Federal de Viçosa, UFV, Viçosa, 111p., 2000.

FREIRE, P. Pedagogia da autonomia - Saberes necessários à prática educativa. São Paulo: Paz e Terra, 37 ed., 148p., 1996.

HARLEY, J. B. Maps, knowledge, and power. In: Cosgrove, Denis \& Daniels, Stephen (org.). The iconography of landscape: Essays on the symbolic representation, design and use of post environments. Cambridge University Press, 1 ed., 162p, 1988. 
HERLIHY, P. H.; KNAPP, G. Maps of, by, and for the peoples of Latin America. In: Human organization. Journal of the Society for Applied Anthropology, v. 62, n. 4, p. 303-314, 2003.

IBGE. Instituto Brasileiro de Geografia e Estatística. (2007). <http://www.ibge.gov.br> LATOUR, B. A esperança de Pandorra: Ensaios sobre a realidade dos estudos científicos. Bauru, SP : EDUSC, 2001, 372p.

LAURIOLA, V. Ecologia global contra diversidade cultural? Conservação da natureza e povos indígenas no Brasil. O Monte Roraima entre Parque Nacional e a Terra Indígena Raposa-Serra do Sol. Revista Ambiente \& Sociedade, v. 5, n. 2, p. 165189, 2003.

LITTLE, P. Gestão territorial em Terras Indígenas: definição de conceitos e proposta de diretrizes. Secretaria de Estado de Meio Ambiente e Recursos Naturais - SEMA-AC, Secretaria Extraordinária dos Povos Indígenas - SEPI-AC e Agência de Cooperação Técnica Alemã (GTZ) no Brasil - GTZ, Rio Branco, Acre, 2006.

MELO, W. F., SILVA, S. S., TAVARES, R. A., FRESCHI, J. M., GAVAZZI, R. A., SILVA, J. F. M., PIYANKO, B., APIWTXA, C. A., BROWN, I. F. Aplicação de dados SRTM, sensoriamento remoto e SIG em etnomapeamento: o caso da Terra Indígena Kampa do Rio Amônia na fronteira Brasil-Acre/Peru-Ucayali. In: Simpósio Brasileiro de Sensoriamento Remoto, INPE, n.13, Florianópolis, SC, 2007. Disponível em: <http://marte.dpi.inpe.br/col/dpi.inpe.br/sbsr@80/2006/11.15.23.05/doc/59495956.pdf $>$ Acesso em 15/06/2010

ORLOVE, B. Mapping reeds and reading maps: the politics of representation in Lake Titicac. American Ethnologist, v. 18, n. 1, p. 3ᄀ38, 1991.

PATAXÓ, R. Encontro com o povo Pataxó de Carmesia. Grupo de Estudos dos Povos Indígenas de Minas Gerais. Universidade Federal dos Vales do Jequitinhonha e Mucuri (UFVJM). Campus I Diamantina, Brazil, 2008.

SCHWARTZMAN, S., ZIMMERMAN, B. Conservation alliances with indigenous peoples of the Amazon. Conservation Biology, v. 19, n. 3, p. 721-727, 2005.

SMITH, D. A. Participatory mapping of community lands and hunting yields among the bugle of Western Panama. Human Organization. Journal of the Society for Applied Anthropology, v. 62, n. 4, p. 332-343, 2003.

THE NATURE CONSERVATION. Etnomapeamento nas comunidades indígenas

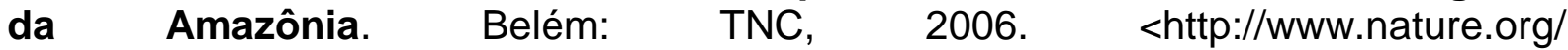
wherewework/southamerica/brasil/work/art16607.html>

VERDEJO M. E. Diagnóstico Rural Participativo. Guia Prático. 1.ed. Brasília: Ministério do Desenvolvimento Agrário, 62 p., 2006. 
VIRGILIO H, BARROS M. V. Z SIG aplicado à caracterização sócio ambiental da reserva indígena Apucarana (PR). Estudos Geográficos, v. 5, n. 1, p. 47-62, 2007. Disponível em: <http://cecemca.rc.unesp.br/ojs/index.php/estgeo>. Acesso em 15/06/2010. 